Behind the Wheel:

What Drives the Effects of Error Handling

\author{
Nicoletta G. Dimitrova \\ Ghent University, Belgium \\ Edwin A. J. van Hooft \\ University of Amsterdam, The Netherlands \\ Cathy van Dyck \\ VU University, Amsterdam, The Netherlands \\ Peter Groenewegen \\ VU University, Amsterdam, The Netherlands
}

Correspondence concerning this article should be addressed to Nicoletta G. Dimitrova, Ghent University, Department of Personnel Management, Work and Organizational Psychology, Henri Dunantlaan 2, 9000 Gent, Belgium

E-mail: nicoletta.dimitrova@ugent.be

Phone: +3292646459 


\begin{abstract}
Existing research comparing error management (a strategy focusing on increasing the positive and decreasing the negative consequences of errors) to error prevention (a strategy focusing on working faultlessly), has identified error management as beneficial for multiple outcomes. Yet, due to various methodological limitations, it is unclear whether the effects previously found are due to error prevention, error management, or both. We examine this in an experimental study with a 2 (error prevention: yes vs. no) x 2 (error management: yes vs. no) factorial design. Error prevention had negative effects on cognition and adaptive transfer performance. Error management alleviated worry and boosted one's perceived self-efficacy. Overall, the results show that error prevention and error management have unique outcomes on negative affect, self-efficacy, cognition, and performance.
\end{abstract}

Keywords: error, negative affect, self-efficacy, cognition, performance 


\section{Behind the Wheel:}

\section{What Drives the Effects of Error Handling}

There has been a considerable interest in understanding how people can deal better with errors because proper error handling can result in learning and growth, whereas poor error handling can cause much harm to oneself and to others. Prior work has identified two error handling strategies: error prevention, which focuses on preventing the occurrence of errors as not to suffer their negative consequences, and error management, which suggests to accept errors as a part of life, and invest one's effort in minimizing their negative consequences (see Frese \& Keith, 2015; Hofmann \& Frese, 2011; Keith \& Frese, 2008).

The assumption explicitly made in most existing work on error handling is that error management is adaptive. This perspective, which we will refer to as the error management advantage perspective has been adopted in most of the error handling literature (e.g., Bell \& Kozlowski, 2008; Chillarege, Nordstrom \& Williams, 2003; Frese, Brodbeck, Heinbokel, Mooser, Schleiffenbaum, \& Thiemann, 1991; Keith \& Frese, 2005; 2008; Van Dyck, Van Hooft, De Gilder, \& Liesveld, 2010). Error management compared to error prevention, has been found to have positive effects on people's affect (e.g., lower frustration and negative affect, Chillarege et al., 2003; stronger emotion control, Keith \& Frese, 2005), motivation (Bell \& Kozlowski, 2008; Wood, Kakebeeke, Debowski, \& Frese, 2000), cognition (e.g., ontask thoughts, Dimitrova, Van Dyck, Van Hooft, \& Groenewegen, 2015; meta-cognition, Bell \& Kozlowski, 2008; Keith \& Frese, 2005), and performance (Bell \& Kozlowski, 2008; Chillarege et al., 2003; Dimitrova et al., 2015; Keith \& Frese, 2005; 2008; Nordstrom, Wendland, \& Williams, 1998).

An alternative is the error prevention disadvantage perspective, which instead focuses on error prevention as being maladaptive (e.g., Loh, Andrews, Hesketh, \& Griffin, 2013). According to this perspective, the effects previously found could have been driven by 
the negative effects of error prevention rather than by the positive effects of error management. Nonetheless, the evidence to substantiate such claims in the error handling literature is currently missing.

A third, synergistic perspective, in line with the high reliability organizations literature, suggests that combining error prevention with error management will be even more adaptive than using either strategy alone, because during most activities people need to balance the two (e.g., Frese \& Keith, 2015; Goodman, Ramanujam, Caroll, Edmondson, Hofmann, \& Sutcliffe, 2011; Weick \& Sutcliffe, 2007). Such a combination can potentially amplify the strengths while minimizing the weaknesses of the two strategies. However, no study to date has investigated whether combining error prevention with error management offers additional benefits over and above error management alone.

The main research question we investigate in the current study is whether it is error prevention, error management, or their combination that drives the effects on affective (i.e., worry), motivational (i.e., self-efficacy), cognitive (i.e., on-task thoughts, negative selfrelated off-task thoughts), and behavioral outcomes (i.e., analogical and adaptive transfer performance). In line with action theory (Frese \& Zapf, 1994), existing error handling studies investigated whether the error framing provided by error prevention and error management impacts affective, cognitive, and behavioral outcomes (for a review see Frese \& Keith, 2015). It remains unclear, however, when is it that error management or error prevention causes these effects. Existing error handling research has been criticized (e.g., Bell \& Kozlowski, 2008; Gully, Payne, Koles, \& Witeman, 2002; Loh et al., 2013; Dimitrova et al., 2015) for exhibiting various methodological limitations (e.g., lack of control groups, use of control groups that differ from the experimental groups on multiple dimensions, and confounding type of practice with type of instructions). In the present study we amend those limitations by using a full-factorial design and by keeping type of practice constant among 
groups. Overall, we aim to contribute to the literature by increasing our understanding of the unique effects of error management and error prevention, as well as of their potential interaction.

\section{Error Handling Strategies}

Errors are potentially avoidable (i.e., not due to some unforeseeable chance agency) and unintended (i.e., not purposeful) deviations, occurring during goal directed action (i.e., errors imply a non-achievement of goals, with achieving the goal as the intention, and the error causing the non-achievement as the deviation; Frese \& Zapf, 1994; Hofmann \& Frese, 2011; Reason, 1990). We define error handling as the process of dealing with errors before and after they have occurred.

Error prevention is a strategy focusing on removing the negative consequences of errors by not allowing them to occur in the first place (Bell \& Kozlowski, 2008; Hofmann \& Frese, 2011; Keith \& Frese, 2005; 2008, Van Dyck, Baer, Frese, \& Sonnentag, 2005). Although intuitively error prevention is not negative per se, solely focusing on prevention has been linked to various negative outcomes, such as hiding errors, lowered learning from errors, negative error cascades, lowered psychological safety within teams, and poor performance (e.g., Edmondson, 1996; 1999; Van Dyck et al., 2005). These findings may contradict common logic, which suggests that preventing errors is good practice. This contradiction might be explained by considering the situation and task. That is, in predictable situations with straightforward tasks, error prevention can be applied and may potentially result in even better outcomes than error management. However, this is not the case in situations in which pure error prevention is impossible because people are dealing with novel and/or relatively complex tasks, which are also the types of situations and tasks predominantly used in error handling research.

The other strategy discussed in the context of error handling is error management and 
focuses on disconnecting the error from its potential negative consequences (Hofmann \& Frese, 2011). It is a strategy that "accepts that errors will occur, and is designed to provide a second layer of defense, specifically one that intends to intercept and rectify the error prior to the accrual of significant negative consequences" (Hofmann \& Frese, 2011, p. 32), while maximizing potential positive consequences (e.g., learning, secondary error prevention). Existing research has shown that in novel situations involving complex tasks error management compared to error prevention has positive effects on affect, motivation, cognition, and performance (e.g., Bell \& Kozlowski, 2008; Chillarege et al., 2003; Dimitrova et al., 2015; Keith \& Frese, 2005; 2008; Wood et al., 2000). A positive association between error management and performance has also been found in well-established situations involving familiar tasks (see Van Dyck et al., 2005).

\section{Affective and Motivational Consequences}

In line with previous research, we focus on worry as an indicator of negative affect (also see Chillarege et al., 2003; Frese et al., 1991; Nordstrom et al., 1998) and self-efficacy as a motivational indicator (e.g., Bell \& Kozlowski, 2008; Carter \& Beier, 2010). Making errors is by default stressful for people (e.g., Reason, 1990), but the way that errors are framed may either be more or less likely to be conducive to stress and worry. Specifically, error management frames errors as neutral occurrences, which if handled properly, do not materialize into negative consequences, and which can be utilized as potentially beneficial learning opportunities. The potential positive effects of errors are often overshadowed by errors causing a lot of negative emotions that people need to deal with (Frese et al., 1991). By becoming more tolerant of errors the emotional turmoil errors cause can be partially minimized. Moreover, making an error is not immediately equated with the potential negative consequences because a person can still trap the error and in this way prevent or lessen its negative consequences, without preventing the error itself (Frese \& Zapf, 1994; 
Hofmann \& Frese, 2011). This sense of control associated with error management is likely to lead to greater experience of self-efficacy.

Error prevention, in contrast, presents errors as negative occurrences that should be prevented, and equates errors with negative consequences. Experiencing negative affect about making errors is a common human response (e.g., Reason, 1990; Zhao, 2011). The realization that one has made an error that could have been avoided and that has potential negative consequences is likely to make most people question their competence or selfefficacy or to worry about the potential consequences of the error. Accordingly, error prevention is likely to result in increased worry and decreased perceived self-efficacy.

Empirical evidence shows that error management results in less frustration and negative affect than error prevention (Chillarege et al., 2003; Frese et al., 1991; Nordstrom et al., 1998). Also, perceived managerial intolerance of errors, which is conceptually similar to error prevention, was positively linked to employees' negative emotions (Zhao, 2011).

Regarding self-efficacy, previous findings have been less conclusive. Error handling was not found to have an effect on task specific self-efficacy (Bell \& Kozlowski, 2008; Gully et al., 2002; Wood et al., 2000), while a study adopting a more general perceived self-efficacy measure, similar to the one we use, found that compared to controls, groups receiving error management instructions experienced greater task self-efficacy (Carter \& Beier, 2010).

\section{Cognitive Consequences}

People have limited cognitive capacity and according to cognitive resource allocation theory mental resources are distributed among on-task thoughts, off-task thoughts, and selfregulation (Kanfer \& Ackerman, 1989). On-task thoughts are defined as attention directed towards a specific task (Smallwood, Obonsawin, \& Heim, 2003), whereas off-task thoughts involve disengaging attention away from goal-directed action (Smallwood, Riby, Heim, \& Davis, 2006). Earlier research has consistently shown that off-task thoughts, especially 
negative self-related off-task thoughts (i.e., thoughts that involve a negative self-evaluation) take away valuable resources people need to focus on a task (e.g., Carver, 1996; Dickhäuser, Buch, \& Dickhäuser, 2011). Self-regulation redirects cognitive resources from off-task thoughts to on-task thoughts. Importantly, cognitive resource allocation theory (Kanfer \& Ackerman, 1989) postulates that the engagement in any of these processes depends on limited attentional resources. Therefore, allocating attentional resources to one activity implies that there will be fewer resources for another. Such reasoning is in line with the work of Keith and Frese (2005) and Bell and Kozlowski (2008) investigating error handling in the context of emotion control.

Similarly to Dimitrova and colleagues (2015) we predict that error management leads to more on-task thoughts because people do not have to constantly question themselves and their ability while working on the task, which makes it easier to stay task focused. When errors are seen as learning opportunities, making an error does not divert attention away from the task as much as when errors are seen as indicators of poor ability. Moreover, making errors signals that a person is failing at prevention, which may lead to questioning of abilities, thus resulting in negative self-focused attention rather than in attention toward the task.

\section{Transfer Performance}

Existing work differentiates between two types of transfer performance: analogical and adaptive transfer performance (see Bell \& Kozlowski, 2008; Keith \& Frese, 2005). Analogical transfer "involves using a familiar problem to solving a problem of the same type" (Ivancic \& Hesketh, 2000, p. 1967), whereas adaptive transfer involves "using one's existing knowledge base to change a learned procedure, or to generate a solution to a completely new problem" (Ivancic \& Hesketh, 2000, p.1968). Regarding error handling and performance, an earlier meta-analysis has shown that receiving error management training rather than other types of training (e.g., step-by-step proceduralized error-prevention training; pure exploratory 
training similar to our control condition) results in better adaptive transfer performance (Keith \& Frese, 2008; also see Bell \& Kozlowski, 2008). These findings were replicated by a recent study using identical type of practice (error-based) and differentiating only between instruction type: error management or error prevention (Dimitrova et al., 2015). Regarding analogical transfer performance, findings about the efficacy of error management instructions are mixed, with some studies finding effects, while others do not (see Dimitrova et al., 2015 and Keith \& Frese, 2008). According to Keith and Frese (2008) the reason for the mixed results might be that "methods that emphasize error free learning and correct procedures for a particular task may be equally effective, as they directly teach the required procedures that are then merely applied to the similar transfer task" (p. 62). Overall, existing findings show that the effectiveness of error management compared to error prevention is more evident for adaptive than for analogical transfer performance (Keith \& Frese, 2008).

\section{Combining Error Prevention and Error Management}

Although it has previously been suggested that in practice both error prevention and error management are necessary (e.g., Hofmann \& Frese, 2011; Van Dyck et al., 2005) we do not yet know whether, how, and when the two error handling strategies combine with one another. The existing theorizing and research on error handling has primarily focused on comparing and contrasting error prevention with error management (e.g., Hofmann \& Frese, 2011; Keith \& Frese, 2005; 2008; Nordstrom et al., 1998). In most situations, however, people use a combination of both, in the sense that they first prefer not to make an error, but if they do, they adapt and focus on dealing with it and hopefully minimize its negative consequences (Hofmann \& Frese, 2011). It is also possible that reminding people of the appropriate steps when dealing with errors provides them with universal and easy cognitive scripts to follow while working on a task. If that is the case, the combination of prevention and management may be more than the sum of its parts: People will not only proactively 
prevent errors, but also constructively handle the errors that could not be prevented, resulting in more beneficial outcomes than either strategy alone. An alternative view, however, could be that people struggle with simultaneously applying the two strategies while learning a new task, which may result in higher cognitive load, inability to cope with the demands of the task, and poor performance.

The current study aims to provide the first empirical investigation clarifying whether error prevention and error management interact (if at all) and how (positively or negatively). In summary, the rationales discussed lead to the following hypotheses and research question: Hypothesis 1: Error management compared to no error management leads to (a) more on-task thoughts, (b) fewer negative self-related off-task thoughts, (c) less worry, (d) higher perceived task self-efficacy, (e) increased post-practice performance (consistent with the error management advantage perspective). Hypothesis 2: Error prevention compared to no error prevention leads to (a) fewer on-task thoughts, (b) more negative self-related off-task thoughts, (c) more worry, (d) lower perceived task self-efficacy, (e) worse post-practice performance (consistent with the error prevention disadvantage perspective). Research question 1: Does combining error management and error prevention lead to better affective, motivational, cognitive or behavioral outcomes than either error prevention or error management alone?

\section{Method}

\section{Participants and Design}

Participants were recruited through flyers distributed in the university cafeteria and through student assistants advertising the study. In total 114 students participated, of whom 48 were men $\left(M_{\text {age }}=21.58 ; S D_{\text {age }}=2.51\right)$ and 66 were women $\left(M_{\text {age }}=22.32 ; S D_{\text {age }}=2.96\right)$. While keeping the gender ratios between groups similar, participants were randomly assigned 
to a 2 (error management instructions: yes vs. no) x 2 (error prevention instructions: yes vs. no) between-participants design. Three participants who indicated participating in a study using the same task before were removed from the sample, because they were familiar with the purpose of the experiment, resulting in a final sample of 111 students (46 men and 65 women; $\left.M_{\text {age }}=22.03 ; S D_{\text {age }}=2.80\right)$.

\section{Procedure}

The experiment lasted about 80 minutes and was presented as a study of the different types of thoughts people have while learning a novel task. As remuneration for their participation, participants received $€ 9$ or course credits.

Simulation task. In the current experiment we used a free-source PC simulation called Train Dispatcher $2.0^{\odot}$ (Signal Computer Consultants, 1997) that has been validated as an appropriate task to study the effects of error handling strategies in prior research

(Dimitrova et al., 2015). In the simulation the participant acts as a train dispatcher whose main goal is to ensure that trains reach their correct end destinations. To move the trains to their exit location the dispatcher uses switches (to switch between tracks) and signals (to direct the trains in the desired direction). The format of brief instructions followed by practice and performance segments is similar to that used in previous studies (e.g., Bell \& Kozlowski, 2008; Dimitrova et al., 2015; Keith \& Frese, 2005).

Task familiarization. All participants received basic instructions outlining the general rules of the simulation. After the brief introduction participants had five minutes to familiarize themselves with the task by guiding a train to its end destination. For that purpose, participants received a handout from the experimenter outlining which train they had to dispatch, what its arrival time was, and what end destination it had to be directed to. Participants who did not manage to guide the train within five minutes were shown by the experimenter the correct procedure. 
Manipulation. After getting familiarized with the task all participants received the manipulation of error handling strategy instructions. A pilot study indicated that the error handling manipulations were understood by participants as intended and were effective in inducing responses consistent with the manipulated strategy. The instructions for the error management only group and the error prevention only group were based on the instructions by Nordstrom et al. (1998), Keith and Frese (2005), Carter and Beier (2010) and Dimitrova et al. (2015). In the error management only group, in line with earlier definitions and research (see Hofmann \& Frese, 2012; Keith \& Frese, 2005), participants read a short text framing errors positively and focusing on maximizing their positive consequences (i.e., learning). Specifically, the error management manipulation stated that "making errors is a natural part of the learning process" (also see Keith \& Frese, 2005 and Dimitrova et al., 2015) and that "when one makes an error it is important to think of how it can be corrected, what can be learned from it, and how what you learn can help you in similar situations in the future". The text also stated that "by successfully handling errors you will learn how to deal with the task more effectively" and "if you try to find the useful information errors provide, you will do better on the task".

Participants in the error prevention only group read a text framing the prevention of errors positively (see Dimitrova et al., 2015; Keith \& Frese, 2005; Nordstrom et al., 1998), saying that "errors should not be allowed to occur during the learning process" and "it is important to think of how you can prevent errors from occurring, what you can do to successfully detect situations possibly leading to errors, and how what you know can help you with similar situations in the future". Participants also read that "by successfully preventing errors you will learn how to deal with the task more effectively" and "if you try to prevent errors from occurring you will do better on the task".

Participants in the mixed error prevention and error management group received a 
combination of the error prevention and error management instructions, which were reworded in a way that stated that both prevention and management are important for performing well on the task. Participants read that they should "first try to prevent errors, but remain open to the possibility that errors will nonetheless occur during learning". Participants next read that "What is important is how you deal with errors and how you can prevent errors in the future. When you make an error it is important to think about how this error can be corrected, what you can learn from it, what you can do to successfully detect situations possibly leading to errors, and how what you know can help you with similar situations in the future so that you prevent similar errors".

Finally, the error neutral group received a neutral text that was comparable in size to the instructions received by the other groups, but contained no information about errors. Specifically, the text mentioned general information on what a train is and the types of trains. While being related to the task because it was discussing trains, none of the information was relevant to the gameplay itself as to avoid influencing participants in any way.

Task practice. Task practice consisted of two ten-minute trials, separated by a two minute break. All participants were given a train schedule which included information on the name of trains, their arrival time, entry point and exit destination. Additionally, during the task practice trials participants had access to the full simulation manual.

\section{Dependent Variables}

With the exception of worry, which was measured after the practice phase of the task, the measurements of self-efficacy, on-task thoughts, and off-task thoughts were administered after the two performance trials. ${ }^{1}$ All answers were given on a 5-point Likert scale ranging from 1 (not at all) to 5 (very much so).

Worry. Participants' experienced worry during the task was measured with two selfdeveloped items "During the task I felt anxious" and "During the task I felt worried" $(\alpha=$ 
.88). These items were based on the state anxiety items of the State-Trait Anxiety Inventory (Spielberger, Gorsuch, Lushene, Vagg, \& Jacobs, 1983; e.g.,"I am worried”), which is among the most used measures of state anxiety and worry (Rose \& Devine, 2014).

Self-efficacy. We measured self-efficacy with five items adapted from Schwarzer and Jerusalem's (1995) Generalized Self-Efficacy Scale that were selected on the basis of relevance to the current content and the context of the task. Items were adjusted to refer to the current context by adding "during the task": "During the task, I was confident that I could deal efficiently with unexpected events", "Thanks to my resourcefulness during the task, I knew how to handle unforeseen situations", "During the task, I could solve most problems if I invested the necessary effort", "During the task, when I was confronted with a problem, I could usually find several solutions", and "During the task, I could usually handle whatever came my way". The adapted scale showed an internal consistency $(\alpha=.87)$ comparable to that found in prior studies (e.g., Schwarzer, Bäßler, Kwiatek, Schröder, \& Zhang, 1997; values range between $\alpha=.81$ and $\alpha=.91$ ).

\section{Cognition.}

On-task thoughts. We used an abbreviated three item on-task thoughts scale adapted from Dimitrova et al. (2015): "I found it easy to concentrate on the task", "I found it easy to keep thinking about what I was supposed to do", and "It was easy to concentrate on what I was doing" $(\alpha=.87)$. The scale showed reliability similar to that found with the original 6item scale in prior research (Dimitrova et al., 2015; Study 1, $\alpha=.87$; Study 2, $\alpha=.89$ ).

Negative self-related off-task thoughts. Negative self-related off-task thoughts were measured with three items previously used by Dickhaüser et al. (2011). The items were: "I thought about how poorly I was doing", "I thought about how dissatisfied I was with my achievement", and "I thought about how unskilled I was in this task" $(\alpha=.87)$.

Post-practice performance. We incorporated measures of performance similar to 
those used by Keith and Frese (2005), Bell and Kozlowski (2008), and Dimitrova et al. (2015). Specifically, participants completed two 10-minute performance trials. Participants were told that the purpose of the performance trials was to show what has been learned during practice and instructed to guide as many trains as possible to their end destinations. Before each trial participants received the train schedule applicable to the trial. The final task score was calculated automatically by the software.

Analogical transfer performance. The first 10-minute performance trial measured analogical transfer and had a level of difficulty comparable to that of the practice session (the number of trains to be dispatched was similar to that during the practice session; the track territory had a similar number of main tracks and entrances/exits). We used the final score automatically calculated at the end of the first performance trial as a measure of analogical transfer performance.

Adaptive transfer performance. Next, after a two-minute break participants started working on the final 10-minute performance trial. The track territory selected for the adaptive transfer trial was much more complex than the track territories presented during all previous trials. Specifically, the number of trains that could potentially be dispatched was almost 4 times higher than that in prior trials. Additionally, the number of tracks was 5 times higher than in previous trials and so was the number of entrances/exits ( 3 times higher). The final performance trial also included a new type of switch that completely changed the behavior of the tracks, as well as tracks that could only be reached by reversing the train. Because the final performance task required adapting and reshaping one's existing knowledge (e.g., using the new switches and reversing trains) and generating solutions to the new problems caused by the multi-track switches, it was used for measuring adaptive rather than analogical transfer performance (see Bell \& Kozlowski, 2008; Keith \& Frese, 2005). The final score as calculated by the program was used as the measure for adaptive transfer 
performance.

\section{Control Variables}

In our analyses, we controlled for participant's sex, age, prior task experience and practice performance. We included sex because we expected that men may overall experience greater self-efficacy while working on the current task than women (also see Bell \& Kozlowski, 2008; Keith \& Frese, 2005). Additionally, earlier research using the same task found that men had higher adaptive transfer performance than women (Dimitrova et al., 2015). Regarding age, previous error handling research has found that younger participants had more on-task and fewer self-related off-task thoughts (Dimitrova et al., 2015) and older participants performed worse on tasks requiring computer proficiency (e.g., Carter \& Beier, 2010; Chillarege et al., 2003). We included prior task experience with similar simulations as a control variable because more experienced participants may be responding differently to the task than less experienced participants (e.g., worrying less or experiencing greater selfefficacy). Additionally, we controlled for participants' practice score performance to account for the possibility that participants emotional, motivational, cognitive, and behavioral responses were affected by how well they performed during the practice phase.

\section{Manipulation Check}

As a manipulation check, we asked participants two statements. One statement was consistent with following error prevention principles and measured whether participants' attempted to be more cautious while working on the task ("During the task I tried to be as cautious as possible"). Another statement was consistent with following error management principles and measured participants' willingness to see errors in a less negative light ("I viewed my errors during the task as surmountable obstacles").

\section{Results}

Manipulation check. Supporting the validity of the manipulation, a 2 (EM: yes vs. 
no) x 2 (EP: yes vs. no) ANOVA yielded a main effect for error prevention on the error prevention statement. Specifically, participants who received error prevention instructions were more likely to say that they tried to be cautious than participants who did not receive error prevention instructions, $F(1,107)=6.64, p=.011, \eta_{\mathrm{p}}^{2}=.06$. Additionally, a second 2 $\mathrm{x} 2$ ANOVA yielded a main effect for error management on the error management statement. Namely, participants who received error management instructions were more likely to perceive their errors during the task as surmountable obstacles than participants who did not receive error management instructions, $F(1,107)=5.70, p=.019, \eta_{\mathrm{p}}^{2}=.05$.

[Place Table 1 about here]

\section{Main Analyses}

Because our dependent variables correlated with each other (see Table 1), we conducted a two-way MANCOVA (controlling for age, sex, prior experience with similar tasks, and practice performance) to account for the relationships between the dependent variables. The overall MANCOVA (using Pillai's trace) analysis only showed significant effects for error prevention on the dependent variables, $V=0.13, F(6,98)=2.47, p=.029$, $\eta_{\mathrm{p}}^{2}=.13$, and for practice task performance, $V=0.42, F(6,98)=11.84, p<.001, \eta_{\mathrm{p}}^{2}=.42$, on the dependent variables (see Table 2 ). No other significant effects were found (all $F$ s $<$ 1.84 , all $p \mathrm{~s}>.098)$.

[Place Table 2 about here]

Separate univariate two-way ANCOVAs showed main effects of error prevention on on-task thoughts, $F(1,103)=5.96, p=.016, \eta_{\mathrm{p}}^{2}=.06$, Cohen's $d=0.46$, negative selfrelated off-task thoughts, $(1,103)=4.63, p=.034, \eta_{p}^{2}=.04$, Cohen's $d=0.42$, and adaptive transfer performance, $F(1,103)=5.06, p=.027, \eta_{\mathrm{p}}^{2}=.05$, Cohen's $d=0.41$. The means as displayed in Table 3 indicate that people who received error prevention instructions experienced fewer on-task thoughts $(M=3.26, S D=1.11)$, more negative self-related off- 
task thoughts $(M=3.22, S D=1.16)$, and performed worse during the adaptive transfer trial $(M=-0.25, S D=0.78)$ than the people who did not receive error prevention instructions $(M=$ 3.71, $S D=0.81, M=2.74, S D=1.14$, and $M=0.10, S D=0.93$, for on-task thoughts, negative self-related off-task thoughts, and adaptive transfer performance, respectively).

Additionally, although the overall MANCOVA did not show any effects of error management, the follow up univariate two-way ANCOVAs showed a significant main effect of error management on worry, $F(1,103)=4.46, p=.037, \eta_{\mathrm{p}}^{2}=.04$, Cohen's $d=0.43$. The means (see Table 3) indicate that people who received error management instructions were less worried while working on the task $(M=2.01, S D=0.94)$ than people who did not receive error management instructions $(M=2.46, S D=1.16)$.

[Place Table 3 about here]

A separate univariate two-way ANCOVA showed a significant interaction effect of error handling instructions on self-efficacy, $F(1,103)=4.08, p=.046, \eta_{p}^{2}=.04$. The means (see Table 3) indicate that participants who received only error management instructions ( $M$ $=3.25, S D=0.76)$ experienced greater self-efficacy than participants who received no error instructions $(M=2.73, S D=0.84)$, only error prevention instructions $(M=2.91, S D=0.94)$, or mixed error management and error prevention instructions $(M=2.72, S D=1.06)$.

Finally, the separate univariate ANCOVAs showed a significant effect of the covariate sex on perceived self-efficacy, with men experiencing greater self-efficacy compared to women, $F(1,103)=4.81, p=.031, \eta_{\mathrm{p}}^{2}=.05$. Additionally, the covariate age had a significant effect on analogical transfer performance, with younger people performing better compared to older people, $F(1,103)=4.12, p=.045, \eta_{\mathrm{p}}^{2}=.04$. Finally, as can be expected, the covariate practice performance showed a significant effect on both analogical, $F(1,103)=65.85, p<.001, \eta_{\mathrm{p}}^{2}=.39$ and adaptive transfer performance, $F(1,103)=13.46$, $p<.001, \eta_{\mathrm{p}}^{2}=.12$. Participants who performed well during the practice phase, were also 
more likely to perform well during the transfer phase. No other significant effects were found for any of the other control variables (all $F \mathrm{~s}<3.13$, all $p \mathrm{~s}>.080$ ).

\section{Discussion}

\section{Theoretical Relevance}

The present study sought to examine what specifically drives the effects of error handling strategy on affective, motivational, cognitive, and behavioral outcomes. We advance the error handling literature by reexamining the error management advantage perspective that has dominated recent error handling research. The current results provide strongest support for the error prevention disadvantage perspective by suggesting that error prevention rather than error management may be driving the previously found effects on cognition and performance. An important implication emerging from this finding is that error prevention during training may be bad for task-focus and performance, rather than error management being good for it. Note that such a conclusion is in line with a recent paper by Loh and colleagues (2013) who similarly suggest that error prevention is more harmful than error management is beneficial. More specifically, Loh and colleagues (2013) found no significant main effect of error management training (EMT) on performance, when comparing it to a control group which received identical information and training (active exploration) and stated that "...the positive effects of error encouragement reported in prior EMT research may actually be attributable to the deleterious impact of error-avoidance training" (p. 444).

Although going against most recent theorizing, which focuses on the benefits of error management for cognition and performance, such a finding is in line with the original action theory framework (Frese \& Zapf, 1994), which advocates error management as an alternative to error prevention, due to the negative effects of error prevention, but not due to the positive effects of error management. An observation of the effects found in earlier work suggests 
that there are bigger differences between groups when error management is compared to error prevention than when error management is compared to pure exploration (see Keith \& Frese's 2008 meta-analysis). Altogether these findings suggest that the conclusions drawn in various earlier studies might have been based on a misinterpretation of the role that error management plays.

Nonetheless, before dismissing the role of error management we should interpret the complete results, which offer us a more valenced picture. The ANCOVAs show that error management instructions lowered the level of worry experienced. Moreover, receiving error management instructions increased people's experienced self-efficacy, but only when error management was not supplemented with error prevention. Thus, two major implications of our findings are that error management does not make people appraise errors in a more positive light but in a less negative light, and that error management influences affect and coping (see Bell \& Kozlowski, 2008; Keith \& Frese, 2005; Nordstrom et al., 1998).

Overall, the picture that emerges from our data suggests that error prevention dampens task-focus and adaptive transfer performance, as well as amplifies negative selfevaluative off-task thoughts, whereas error management dampens worry and boosts one's perceived self-efficacy. The current pattern of findings shows that instead of affecting the same variables, error management and error prevention have unique outcomes. Thus, our results highlight that there may be a grain of truth in both the error prevention disadvantage perspective and the error management advantage perspective. However, which perspective is valid seems to depend on what outcome is being investigated. An important theoretical implication of these findings is that the two error handling strategies appear to be operating through separate pathways. Error prevention, influences people's cognition, in terms of their task-related and task-unrelated thoughts, and their actual behavior, in terms of adaptive transfer performance. Error management, in contrast, influences coping by dampening 
negative emotion, as evidenced by less worry. The present study then contributes to existing theorizing by identifying those pathways and offering an explanation to what error prevention and error management actually have impact on.

\section{Limitations and Suggestions for Future Research}

Although our study offers novel and valuable findings, some limitations should be addressed. First, in line with prior theorizing and research (Chillarege et al., 2003; Frese et al., 1991; Nordstrom et al., 1998), we investigated only negative affect. Future research should test whether error management can increase coping by both reducing negative affect and increasing positive affect, whereas error prevention can decrease positive affect. More generally, future research should investigate a broader range of potential outcomes of error management and error prevention, with specific attention to testing the boundary conditions of our effects (e.g., in terms of task settings, task novelty). In addition, further research is needed to clarify if and under what conditions error prevention can have positive rather than negative effects.

Second, due to the timing of measurements - all measurements with the exception of worry were administered after participants completed the two performance trials - our design did not allow for properly testing potential mediation effects of cognitive resource allocation and self-efficacy on performance. Previous research shows that the effects of error handling on performance go through affective, motivational, and cognitive pathways (e.g., Bell \& Kozlowski, 2008; Dimitrova et al., 2015; Keith \& Frese, 2005). In the abovementioned studies affect, motivation, and cognition were always measured during or immediately after the practice phase. It is likely that people's responses change during the course of practicing a task. Such a change is evidenced by the finding that participant's frustration levels are similar immediately after practice, but after the performance phase, participants receiving error prevention training show greater frustration than participants receiving error 
management training (Frese et al., 1991; however, note that in this study error management instructions were paired with exploratory training and error prevention instructions were paired with errorless training). Additionally, further supporting this line of reasoning, the correlations found in a previous study (Dimitrova et al., 2015) between on-task thoughts during practice and analogical and adaptive transfer performance were positive, whereas in the present study, no relationship was found between post-practice on-task thoughts and performance. We recommend that future research more thoroughly investigates changes in affect, motivation, and cognition during and after the practice and performance phases so as to clarify if and how stable effects are over time. Additionally, it will be interesting to test whether the same effects as reported here emerge when incorporating measures immediately after practice.

Third, our choice of task may have negatively affected motivation as the task was not focused on skills participants necessarily wanted or needed to learn for a specific purpose going beyond the experiment itself. During training in organizations there are more incentives for learning new skills, whereas the task we used in our experiment could have been seen by participants as unrelated to their goals, resulting in general amotivation. In fact, amotivation offers a compelling explanation for finding no correlations between performance and on-task thoughts or negative self-related off-task thoughts. Experiencing more negative self-related off-task thoughts suggests that a person is invested in the task. Similarly, being focused (more on-task thoughts) implies that the person is motivated to work on the task. Consequently, our findings might indicate that participants were having a relatively low motivation throughout the study. Additionally, the task we used has certain characteristics (i.e., learning and fast decision-making required, timed) that may limit its generalizability. We encourage replicating the current study in naturalistic organizational settings with working samples involved in tasks with varied characteristics in order to test the external 
validity of our findings.

Finally, although our results did not provide much support for combining error prevention and error management, we are convinced that more research is necessary. Life requires us to both prevent and manage errors, but it is possible that most people are not particularly good at doing both at the same time and may thus engage in serial error prevention and error management (switching when necessary). Future work should shed light on individuals' serial and simultaneous application of error prevention and error management strategies. Additionally, the combination of error prevention and error management strategies poses important questions for organizations. According to Weick and Sutcliffe (2007) an organization that is highly reliable is capable of both preventing and managing errors. Yet, it is unclear if the people within an organization are preventing and managing errors simultaneously during every task. Also, it is unclear if teams perform better when all members of a team focus on prevention, on management, or on a combination of the two. A related question is whether it is useful to have some members within a team focus on error prevention, while others focus on error management aspects. Additionally, it would be worthwhile investigating what is the best proportion of people preventing and managing errors within a team, and if this proportion is influenced by context and task specifics (e.g., in R\&D departments it may be good to have a majority of error management strategy people and a minority of error prevention strategy people to ground them while working on creative tasks). Finally, future research should seek to examine whether and how instructions interact with people's natural error handling strategy.

\section{Practical Relevance}

Although the primary focus of the present study was mainly theoretical integration, clarification, and extension, the present results offer some valuable practical implications. First, including error prevention in training appears to be detrimental for thinking and 
adaptive transfer performance. Accordingly, the overly common training using prevention instructions should be reconsidered as a good manner of learning new information. By instructing people to prevent errors, everything that subsequently goes wrong activates negative self-related off-task thoughts, simultaneously lowering on-task thoughts. Second, providing error management instructions can positively influence people's coping abilities by helping them to lower their worry and increase their perceived self-efficacy when dealing with difficulties. Overall, our recommendation for people and organizations is to minimize the use of error prevention during new skill development due to its harmful effects on cognition and performance. Instead, the alternative of using error management instructions is to be preferred because it nurtures people's coping capabilities. 


\section{References}

Bell, B. S., \& Kozlowski, S. W. J. (2008). Active learning: Effects of core training design elements on self-regulatory processes, learning, and adaptability. Journal of Applied Psychology, 93, 296-316. doi: 10.1037/0021-9010.93.2.296

Carter, M., \& Beier, M. E. (2010). The effectiveness of error management training with working-aged adults. Personnel Psychology, 63, 641-675. doi: 10.1111/j.17446570.2010.01183.x

Carver, C. S. (1996). Cognitive interference and the structure of behavior. In I. G. Sarason, G. R. Pierce, \& B. R. Sarason (Eds.), Cognitive interference: Theories, methods, and findings (pp. 25-45). Mahwah, NJ: Erlbaum.

Chillarege, K. A., Nordstrom, C. R., \& Williams, K. B. (2003). Learning from our mistakes: Error management training for mature learners. Journal of Business and Psychology, 17, 369-385. doi: 10.1023/A:1022864324988

Dickhaüser, C., Buch, S. R., \& Dickhaüser, O. (2011). Achievement after failure: The role of achievement goals and negative self-related thoughts. Learning and Instruction, 21, 152162. doi: 10.1016/j.learninstruc.2010.01.002

Dimitrova, N. G., Van Dyck, C., Van Hooft, E. A. J., \& Groenewegen, P. (2015). Don't fuss, focus: The mediating effect of on task thoughts on the relationship between error approach instructions and task performance. Applied Psychology: An International Review, 64, 599-624. doi: 10.1111/apps.12029

Edmondson, A. C. (1996). Learning from mistake is easier said than done: Group and organizational influences on the detection and correction of human error. Journal of Applied Behavioral Science, 32, 5-28. doi: 10.1177/0021886396321001

Edmondson, A. C. (1999). Psychological safety and learning behavior in work teams. Administrative Science Quarterly, 44, 350-383. doi: 10.2307/2666999 
Frese, M., Brodbeck, F., Heinbokel, T., Mooser, C., Schleiffenbaum, E., \& Thiemann, P. (1991). Errors in training computer skills: On the positive function of errors. HumanComputer Interaction, 6, 77-93.doi: 10.1207/s15327051hci0601_3

Frese, M., \& Keith, N. (2015). Action errors, error management, and learning in organizations. Annual Review of Psychology, 66, 661-687. doi: 10.1146/annurev-psych010814-015205

Frese, M. \& Zapf, D. (1994). Action as the core of work psychology: A German approach. In Harry C. Triandis, Marvin D. Dunette and Leatta M. Hough (Eds.), Handbook of industrial and organizational psychology (Vol. 4, pp. 271-340). Palo Alto: Consulting Psychologists Press.

Goodman, J. S., Ramanujam, R., Caroll, J. S., Edmondson, A. C., Hofmann, D. A., \& Sutcliffe, K. M. (2011). Organizational errors: Directions for future research. Research in Organizational Behavior, 31, 151-176. doi: 10.1016/j.riob.2011.09.003

Gully, S. M., Payne, S. C., Koles, K. L. K., \& Whiteman, J. K. (2002). The impact of error training and individual differences on training outcomes: An attribute-treatment interaction perspective. Journal of Applied Psychology, 87, 143- 155. doi: 10.1037/00219010.87 .1 .143

Hofmann, D. A., \& Frese, M. (Eds.). (2011). Errors in organizations. New York: Routledge. Ivancic, K., \& Hesketh, B. (2000). Learning from error in a driving simulation: Effects of driving skill and self-confidence. Ergonomics, 43: 1966-1984. doi: $10.1080 / 00140130050201427$

Kanfer, R., \& Ackerman, P. L. (1989). Motivation and cognitive abilities: An integrative/ aptitude-treatment interaction approach to skill acquisition. Journal of Applied Psychology, 74, 657-690. doi: 10.1037/0021-9010.74.4.657

Keith, N., \& Frese, M. (2005). Self-regulation in error management training: Emotion control 
and metacognitive activity as mediators of performance effects. Journal of Applied Psychology, 90, 677-691. doi: 10.1037/0021-9010.90.4.677

Keith, N., \& Frese, M. (2008). Effectiveness of error management training: A meta-analysis. Journal of Applied Psychology, 93, 59-69. doi: 10.1037/0021-9010.93.1.59

Loh, V., Andrews, S., Hesketh, B., \& Griffin, B. (2013). The moderating effect of individual differences in error-management training: Who learns from mistakes? Human Factors, 55, 435-448. doi: 10.1177/0018720812451856

Nordstrom, C. R., Wendland, D., \& Williams, K. B. (1998). “To err is human”: An examination of the effectiveness of error management training. Journal of Business and Psychology, 12, 296-282. doi: 10.1023/A:1025019212263

Reason, J. (1990). Human error. Cambridge, NY: Cambridge University Press.

Rose, M., \& Devine, J. (2014). Assessment of patient-reported symptoms of anxiety. Dialogues in Clinical Neuroscience, 16, 197-211. PMCID: PMC4140513

Schwarzer, R., Bäßler, J., Kwiatek, P., Schröder, K., \& Zhang, J. X. (1997). The assessment of optimistic self-beliefs: Comparison of the German, Spanish, and Chinese versions of the General Self-Efficacy Scale. Applied Psychology: An International Review, 46 , 6988. doi: 10.1111/j.1464-0597.1997.tb01096.x

Schwarzer, R., \& Jerusalem, M. (1995). Generalized Self-Efficacy scale. In J. Weinman, S. Wright, \& M. Johnston, Measures in health psychology: A user's portfolio. Causal and control beliefs (pp. 35-37). Windsor, UK: NFER-NELSON.

Signal Computer Consultants. (1997). Train Dispatcher (Version 2.0). Retrieved from http://www.signalcc.com/train2/td2freeware.html

Smallwood, J., Obonsawin, M., \& Heim, D. (2003). Task-unrelated thought: The role of distributed processing. Consciousness \& Cognition, 12, 169-189. doi: 10.1016/S10538100(02)00003-X 
Smallwood, J., Riby, L, Heim, D., \& Davies, J. B. (2006). Encoding during the attentional lapse: Accuracy of encoding during the semantic sustained attention to response task. Consciousness \& Cognition, 15, 218-231. doi: 10.1016/j.concog.2005.03.003

Spielberger, C. D., Gorsuch, R. L., Lushene, R., Vagg, P. R., \& Jacobs, G. A. (1983). Manual for the State-Trait Anxiety Inventory. Palo Alto, CA: Consulting Psychologists Press.

Van Dyck, C., Frese, M., Baer, M., \& Sonnentag, S. (2005). Organizational error management culture and its impact on performance: A two-study replication. Journal of Applied Psychology, 90, 1228-1240. doi: 10.1037/0021-9010.90.6.1228

Van Dyck, C., Van Hooft, E. A. J., De Gilder, T. C., \& Liesveld, L. C. (2010). Proximal antecedents and correlates of adopted error approach: A self-regulatory perspective. Journal of Social Psychology, 150, 428-451. doi: 10.1080/00224540903366743

Weick, K. E., \& Sutcliffe, K. M. (2007). Managing the unexpected: Resilient performance in an age of uncertainty. San Francisco: Jossey-Bass.

Wood, R. E., Kakebeeke, B. M., Debowski, S., \& Frese, M. (2000). The impact of enactive exploration on intrinsic motivation, strategy, and performance in electronic search. Applied Psychology: An International Review, 49, 263-283. doi: 10.1111/14640597.00014

Zhao, B. (2011). Learning from errors: The role of context, emotion, and personality. Journal of Organizational Behavior, 32, 435-463. doi: 10.1002/job.696 


\section{Footnotes}

${ }^{1}$ Our questionnaires also included measures of general off-task thoughts and ego depletion. For reasons of parsimony these were not included in the manuscript. No significant main or interaction effects were found for general off-task thoughts and ego depletion, and inclusion of these measures did not alter the results for the overall MANCOVA. 
Table 1

\section{Correlations Between Study Variables}

\begin{tabular}{|c|c|c|c|c|c|c|c|c|c|c|c|c|c|}
\hline & Variable & 1 & 2 & 3 & 4 & 5 & 6 & 7 & 8 & 9 & 10 & 11 & 12 \\
\hline & Independent variables: & & & & & & & & & & & & \\
\hline 1 & EM (No/Yes) & - & & & & & & & & & & & \\
\hline \multirow[t]{2}{*}{2} & $\mathrm{EP}(\mathrm{No} / \mathrm{Yes})$ & -.03 & - & & & & & & & & & & \\
\hline & Control variables: & & & & & & & & & & & & \\
\hline 3 & Sex & -.03 & -.01 & - & & & & & & & & & \\
\hline 4 & Age & .04 & -.03 & .12 & - & & & & & & & & \\
\hline 5 & Prior experience with similar tasks & $.22^{*}$ & -.02 & -.06 & .06 & - & & & & & & & \\
\hline \multirow[t]{2}{*}{6} & Practice performance & .00 & -.01 & -.07 & $-.21 *$ & -.07 & - & & & & & & \\
\hline & Dependent variables: & & & & & & & & & & & & \\
\hline 7 & Worry & $-.21^{*}$ & .03 & .10 & .14 & -.07 & -.16 & - & & & & & \\
\hline 8 & Perceived self-efficacy & .10 & -.10 & $-.20 *$ & -.05 & .09 & -.15 & $-.37_{* * *}^{*}$ & - & & & & \\
\hline 9 & On-task thoughts & .14 & $-.23^{*}$ & -.05 & -.01 & .01 & -.13 & $-.40^{* * *}$ & $.54^{* * * *}$ & - & & & \\
\hline 10 & Negative self-related off-task thoughts & -.06 & $.20^{*}$ & .01 & -.02 & -.05 & .11 & $.35^{* * *}$ & $-.48^{* * *}$ & $-.40^{* * *}$ & - & & \\
\hline 11 & Analogical transfer performance & -.05 & -.02 & -.11 & $-.27^{* *}$ & -.05 & $.64 * * *$ & -.17 & -.14 & -.06 & .11 & - & \\
\hline 12 & Adaptive transfer performance & -.03 & $-.20^{*}$ & -.01 & -.08 & -.07 &. $.35^{* * *}$ & -.16 & -.00 & .07 & .06 & $.44 * * *$ & - \\
\hline
\end{tabular}

Note. $N=111 . \mathrm{EM}=$ Error management: $0=$ No and $1=$ Yes. $\mathrm{EP}=$ Error prevention: $0=$ No and $1=$ Yes. Sex: $0=$ man and $1=$ woman. The scores for practice, analogical and adaptive transfer performance are standardized in line with the recommendations by Bell and Kozlowski (2008). All tests are two-tailed.

$* p<.05 . * * p<.01 . * * * p<.001$ 
Table 2

Results MANCOVA and Univariate ANCOVAs

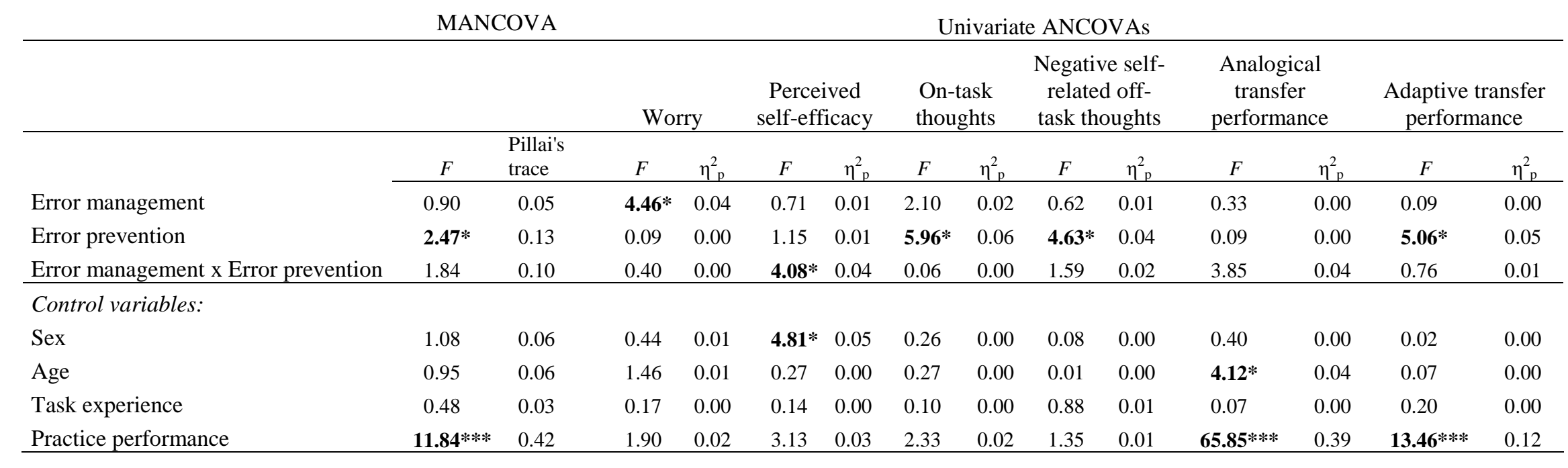

Note. Error management: $0=$ No and $1=$ Yes. Error prevention: $0=$ No and $1=$ Yes. Sex: $0=\operatorname{man}$ and $1=$ woman. $F$ values significant at $p<.05$ are shown in bold. $* p<.05 . * * * p<.001$ 
Table 3

Means and Standard Deviations for all Study Variables per Group

\begin{tabular}{|c|c|c|c|c|}
\hline & $\begin{array}{l}\text { Error Management } \\
\text { Only } \\
\text { (EM: yes; EP: no) } \\
N=28\end{array}$ & $\begin{array}{l}\text { Error Prevention } \\
\text { Only } \\
\text { (EM: no; EP: yes) } \\
N=28\end{array}$ & $\begin{array}{l}\text { Combined Error } \\
\text { Prevention and } \\
\text { Error Management } \\
\text { (EM: yes; EP: yes) } \\
\quad N=26\end{array}$ & $\begin{array}{c}\text { Error Neutral } \\
\text { (EM: no; EP: no) } \\
N=27\end{array}$ \\
\hline & $\mathrm{M}(\mathrm{SD})$ & $\mathrm{M}(\mathrm{SD})$ & $\mathrm{M}(\mathrm{SD})$ & $\mathrm{M}(\mathrm{SD})$ \\
\hline \multicolumn{5}{|l|}{ Control variables: } \\
\hline Age & $22.00(3.04)$ & $21.61(2.50)$ & $22.30(2.80)$ & $22.22(2.93)$ \\
\hline Task experience & $2.28(1.16)$ & $1.75(1.27)$ & $2.04(1.09)$ & $1.59(0.89)$ \\
\hline Practice performance & $-0.08(0.94)$ & $-0.09(1.11)$ & $-0.05(0.80)$ & $-0.03(0.86)$ \\
\hline \multicolumn{5}{|l|}{ Dependent variables: } \\
\hline Worry & $2.03(0.93)_{\mathrm{a}}$ & $2.54(1.30)_{b}$ & $1.98(0.96)_{\mathrm{a}}$ & $2.37(1.01)_{\mathrm{b}}$ \\
\hline Perceived self-efficacy & $3.25(0.76)_{\mathrm{a}}$ & $2.91(0.94)_{b}$ & $2.72(1.06)_{b}$ & $2.73(0.84)_{b}$ \\
\hline On-task thoughts & $3.82(0.80)_{a}$ & $3.11(1.12)_{\mathrm{b}}$ & $3.41(1.10)_{\mathrm{b}}$ & $3.59(0.82)_{a}$ \\
\hline Negative self-related off-task thoughts & $2.55(1.10)_{\mathrm{a}}$ & $3.15(1.27)_{\mathrm{b}}$ & $3.28(1.05)_{\mathrm{b}}$ & $2.95(1.16)_{\mathrm{a}}$ \\
\hline Analogical transfer performance & $-0.22(0.54)$ & $-0.18(0.77)$ & $-0.01(1.11)$ & $0.10(0.88)$ \\
\hline Adaptive transfer performance & $0.00(0.74)_{\mathrm{a}}$ & $-0.29(0.75)_{b}$ & $-0.20(0.83)_{b}$ & $0.22(1.10)_{\mathrm{a}}$ \\
\hline
\end{tabular}

Note. $N=111 . \mathrm{EM}=$ Error management. $\mathrm{EP}=$ Error prevention. The scores for practice, analogical and adaptive transfer performance are standardized in line with the recommendations by Bell and Kozlowski (2008). Cell means that do not share subscripts were significantly different from each other at $p<.05$ in the univariate two-way ANCOVAs. 